\section{Maternal dietary loads of alpha-tocopherol increase synapse density and glial synaptic coverage in the hippocampus of adult offspring}

\author{
S. Salucci, ${ }^{1}$ P. Ambrogini, ${ }^{2}$ D. Lattanzi, \\ M. Betti, ${ }^{2}$ P. Gobbi, ${ }^{1}$ C. Galati, ${ }^{2}$ F. Galli, ${ }^{3}$ \\ R. Cuppini, ${ }^{2}$ A. Minelli ${ }^{2}$ \\ 'Department of Earth, Life and \\ Environmental Sciences (DiSTeVA), \\ Section of Morphology, Carlo Bo \\ University of Urbino \\ 2Department of Earth, Life and \\ Environmental Sciences (DiSTeVA), \\ Section of Physiology, Carlo Bo \\ University of Urbino \\ ${ }^{3}$ Department of Internal Medicine, \\ Section of Applied Biochemistry \\ and Nutritional Sciences, University \\ of Perugia, Italy
}

\section{Abstract}

An increased intake of the antioxidant $\alpha$ Tocopherol (vitamin E) is recommended in complicated pregnancies, to prevent free radical damage to mother and fetus. However, the anti-PKC and antimitotic activity of $\alpha$ Tocopherol raises concerns about its potential effects on brain development. Recently, we found that maternal dietary loads of $\alpha$ Tocopherol through pregnancy and lactation cause developmental deficit in hippocampal synaptic plasticity in rat offspring. The defect persisted into adulthood, with behavioral alterations in hippocampus-dependent learning. Here, using the same rat model of maternal supplementation, ultrastructural morphometric studies were carried out to provide mechanistic interpretation to such a functional impairment in adult offspring by the occurrence of long-term changes in density and morphological features of hippocampal synapses. Higher density of axo-spinous synapses was found in CA1 stratum radiatum of $\alpha$ Tocopherol-exposed rats compared to controls, pointing to a reduced synapse pruning. No morphometric changes were found in synaptic ultrastructural features, i.e., perimeter of axon terminals, length of synaptic specializations, extension of bouton-spine contact. Gliasynapse anatomical relationship was also affected. Heavier astrocytic coverage of synapses was observed in Tocopherol-treated offspring, notably surrounding axon terminals; moreover, the percentage of synapses contacted by astrocytic endfeet at bouton-spine inter- face (tripartite synapses) was increased.

These findings indicate that gestational and neonatal exposure to supranutritional Tocopherol intake can result in anatomical changes of offspring hippocampus that last through adulthood. These include a surplus of axo-spinous synapses and an aberrant gliasynapse relationship, which may represent the morphological signature of previously described alterations in synaptic plasticity and hippocampus-dependent learning.

\section{Introduction}

$\alpha$-Tocopherol ( $\alpha-T)$, the main form of vitamin $\mathrm{E}$ in mammal organisms, is a potent fatsoluble antioxidant and scavenger of hydroperoxyl radicals formed during the lipid peroxidation chain reaction. However, $\alpha-\mathrm{T}$ also possess alternative, non-antioxidant, functions ${ }^{1}$ that occur through the regulation of signaling molecules and the transcriptional control of groups of genes involved in key cellular events such as cell cycle progression and apoptosis, mitochondrial function, xenobiotic and lipid metabolism, etc. ${ }^{2-4}$ Protein kinase C (PKC) appears to be central to vitamin E signaling; ; $^{2-6}$ in fact, $\alpha$-T can potently inhibit PKC activity, as documented in various in vitro model systems ${ }^{5-7}$ and in vivo in both the developing ${ }^{8}$ and adult brain. ${ }^{9} \alpha$-T is regarded as an important molecule during fetal and early postnatal life, playing fundamental roles in protecting the developing organism against oxidative stress. In fact, free radicals have been indicated as causative agents of pregnancy-related disorders, such as preeclampsia and maternal diabetes, inducing serious complications in both the mother and fetus. ${ }^{10,11}$ Blood $\alpha$-T concentrations are reduced in abnormal pregnancies; ${ }^{11-13}$ moreover, preclinical in vitro evidence pointed to a potential therapeutic role of vitamin $\mathrm{E}$ in reducing placental oxidative stress induced by nicotine. ${ }^{14}$ On the bases of this rationale, an increase of vitamin $\mathrm{E}$ intake is recommended in complicated pregnancy to prevent free radical damage to the fetus. ${ }^{15-18}$ However, beneficial effects of vitamin $\mathrm{E}$ in risky pregnancy are currently debated, and the anti-PKC and antimitotic activity of $\alpha$-T raises important caveat against an indiscriminate use of vitamin $\mathrm{E}$ in gestation because of its potential effects on brain development. In fact, PKC plays a pivotal role in brain maturation, ${ }^{19-2}$, and alterations in its activity and/or expression levels have been proposed as the mechanism underlying the neuroteratogenicity of several chemical or physical agents. ${ }^{22-25}$

In previous works, we found that maternal supranutritional dietary intake of $\alpha$-T through pregnancy and lactation in rats markedly
Correspondence: Dr. Andrea Minelli, Department of Earth, Life and Environmental Sciences (DiSTeVA), Section of Physiology, University of Urbino Carlo Bo, via Cà le Suore 2, 61029 Urbino, Italy.

Tel. +39.0722 .304252 - Fax: +39.0722 .304226 .

E-mail: andrea.minelli@uniurb.it

Key words: Vitamin E, CA1 stratum radiatum, axo-spinous synapses, glia-synapse relationship, tripartite synapses, morphometry, electron microscopy.

Conflict of interests: the authors declare no conflict of interest, having no commercial relationships to products or companies related to the subject matter of the article.

Contributions: all authors gave substantial contributions to the conception and design of the work and to the acquisition, analysis, and interpretation of data.

Funding: Carlo Bo University of Urbino, Italy.

Received for publication: 11 December 2013. Accepted for publication: 12 March 2014.

This work is licensed under a Creative Commons Attribution NonCommercial 3.0 License (CC BYNC 3.0).

(C) Copyright S. Salucci et al., 2014

Licensee PAGEPress, Italy

European Journal of Histochemistry 2014; 58:2355 doi:10.4081/ejh.2014.2355

depresses PKC activity also reducing synaptic long-term potentiation (LTP) in developing hippocampus. ${ }^{8}$ Importantly, a deficit in hippocampal synaptic plasticity was produced that persisted in adulthood, accompanied by alterations in hippocampus-dependent learning performances. ${ }^{8,26}$ In particular, adult rats born to tocopherol-supplemented dams performed less efficiently in spatial learning tasks, but displayed improved contextual fear conditioning, thus showing that two kinds of hippocampus-dependent learning abilities display differential sensitivity to the same manipulation of early nutritional environment. ${ }^{26}$.

Collectively, these findings indicated that exposing rats to an excess of $\alpha$-T during development can lead to long-term electrophysiological and neurobehavioral consequences in adulthood, i.e., when tocopherol intake and $\alpha$ T brain accumulation, as well as PKC activity, have returned to normal levels. ${ }^{8}$ The possibility that adult offspring of tocopherol-supplemented dams may carry brain structural changes that could help explaining functional and behavioral alterations in adulthood, remains unexplored.

Here, using the very same rat model of maternal dietary $\alpha$-T supplementation, 
anatomical studies were carried out at the electron microscopy level in order to investigate, in adult offspring, possible changes in density and morphological features of asymmetric, thus excitatory and presumably glutamatergic, ${ }^{27}$ axo-spinous synapses within hippocampal CA1 stratum radiatum, i.e., the brain region where we have previously found a marked reduction of LTP induction ${ }^{8}$ and the area involved in mediating hippocampus-related learning abilities. ${ }^{28,29}$

\section{Materials and Methods}

\section{Animals}

Sprague-Dawley rats (Charles River, Calco, LC, Italy) were used in this study. Animal care and experimentations were performed in compliance with the Italian laws on animal experimentation and with the guidelines of the local Ethical Committee of the University of Urbino Carlo Bo. Virgin female rats (200-250 g) were housed individually with free access to food and water and maintained at a room temperature of $21^{\circ} \mathrm{C}$ with 12 -h light/dark cycle (light on at 6 a.m. and off at 6 p.m.). Dams were randomly divided into two groups: a control group (CTRL; $n=4$ ), fed a commercial standard diet, and a supplemented group (TREAT; $n=4$ ), fed a diet highly enriched in the natural RRR- $\alpha$ tocopherol form (15 $\mathrm{g} / \mathrm{kg}$ pellet; Laboratori Dottori Piccioni, Gessate, MI, Italy), as previously described. ${ }^{8}$ Briefly, $\alpha$-T-enriched diet was administered constantly from 2 weeks before mating throughout pregnancy and lactation. After weaning, pups were separated from mothers and fed ad libitum standard diet. Supplementation protocol did not affect course of pregnancy, newborn survival rate, weight of pups, and litter size, and no obvious teratogenic effects were observed in TREAT newborn. No significant intergroup difference in ponderal growth as well as in the timing of major maturational steps was found, neither obvious abnormalities were observed in older pups. For the present experimental purposes, CTRL and TREAT offspring (6 rats for each group, from 3 different litters) were sacrified at adult age (postnatal day 60, P60).

\section{Tissue preparation}

Rats were deeply anesthetized with sodium pentothal (45 mg/kg) and perfused through the ascending aorta with physiological saline followed by phosphate buffer (PB, $0.1 \mathrm{M} ; \mathrm{pH}$ 7.4) containing $3 \%$ paraformaldehyde and $2 \%$ glutaraldehyde. Brains were removed and postfixed in the same fixative for $12 \mathrm{~h}$, then hippocampi were excised and cut with a vibratome into $30-\mu \mathrm{m}$ thick, parasagittal sections. Tissue preparation for electron microscopy inspection was done as previously described. ${ }^{30,31}$ Briefly, hippocampal sections were postfixed in $2.5 \%$ glutaraldehyde in $\mathrm{PB}$, and then for $1 \mathrm{~h}$ in $\mathrm{OsO}_{4} 1 \%$. After dehydration in graded series of ethanol, sections were cleared in propylene oxide, flat-embedded in Epon-Spurr between acetate sheets (Aclar; Ted Pella, Redding, CA, USA), and polymerized at $60^{\circ} \mathrm{C}$ for $72 \mathrm{~h}$. Embedded sections were examined under a dissecting microscope, and hippocampal areas containing CA1 subfield were excised with a razor blade and mounted on cured resin pyramidal blocks utilizing a cyanoacrylic glue. From each sample, one semithin $(1 \mu \mathrm{m})$ section was cut with a Reichert ultramicrotome and mounted on glass slides for light microscopic inspection. Ultrathin (70-100 nm-thick) sections were cut either from the surface or from the edge (i.e., perpendicular to plane section), counterstained with uranyl acetate and lead citrate, and examined with a Philips CM 10 electron microscope.

\section{Ultrastructural studies}

All data were collected from the hippocampal CA1 stratum radiatum. Identification of neuropilar profiles (presynaptic axon terminals, dendritic spines, astrocytic processes) was based on established morphological and morphometrical criteria. ${ }^{32}$ Counts of synaptic neuronal profiles and astrocytic processes were performed in single plane ultrathin sections. In each rat from the two experimental groups ( $n=6$ for each group), 70 squared areas (100 $\mu \mathrm{m}^{2}$ ) from different mesh nickel grids were scanned at the electron microscope, and all asymmetric, axo-spinous synaptic profiles were sampled for quantitative morphometric analyses. Neuronal and astrocytic profiles were measured in a semiautomatic mode, using the Image J software for image analysis. All the counts and morphometric analyses have been performed by two distinct observers (SS and $\mathrm{AP}$ ).

\section{Data analysis and statistics}

In each scanned area from CA1 stratum radiatum of both CTRL and TREAT rats, the density of axo-spinous synapses was calculated as number of synapses per $\mu^{2}$. In all synapses, we measured: i) the perimeter (in $\mathrm{nm}$ ) of presynaptic axon terminal and dendrit-

Table 1. Basic morphometric features of axo-spinous CA1 synapses.

\begin{tabular}{lcc} 
& CTRL & TREAT \\
Perimeter of axon terminals $(\mathrm{nm})$ & $1691.0 \pm 57.4$ & $1640.4 \pm 51.0$ \\
Length of synaptic specializations $(\mathrm{nm})$ & $235.8 \pm 5.5$ & $239.3 \pm 2.2$ \\
\hline Number of synaptic specialization per terminal & $1.10 \pm 0.03$ & $1.08 \pm 0.01$ \\
Bouton-spine membrane interface extension $(\mathrm{nm})$ & $353.3 \pm 19.8$ & $342.7 \pm 7.7$ \\
\hline
\end{tabular}

CTRL, age-matched control animals; TREAT, tocopherol-supplemented mothers. Data are presented as mean values \pm SEM; Student's t-test; all P>0.05. ic spine profiles; ii) the number and length (in $\mathrm{nm}$ ) of postsynaptic specializations; iii) the extension (in $\mathrm{nm}$ ) of bouton-spine contact. All glial profiles that were in direct apposition to pre- and/or postsynaptic profiles (perisynaptic glial processes) were also counted. Perisynaptic glial processes were classified in two distinct sets: i) processes that contacted axon terminals or dendritic spines, but were located at some distance from bouton-spine interface (extrasynaptic glia), and ii) processes contacting the axo-spinous synaptic junction (intrasynaptic glia), thus directly facing the cleft, i.e., the very site of neurotransmitter release. The length (in $\mathrm{nm}$ ) of membrane apposition between glial processes and pre/postsynaptic profiles was measured, and the ratio between this glia-covered length and the total perimeter of axon terminal and spine contours (after subtracting the length of boutonspine contact) was calculated. Finally, the percentage of synapses contacted by intrasynaptic glia over the total synapses was calculated. Visual details on the methodological approach used for morphometric analyses are given in Figure 1.

For all morphometric indices considered, average values were calculated in each single scanned area and filed for further statistical analyses. All data are presented as mean \pm standard deviation of the mean (SEM). Student's $t$-test was applied for statistical analyses to compare results obtained in CTRL vs TREAT rats; $\mathrm{P}=0.05$ was considered as significance threshold.

\section{Results}

In adult rats born to dams supplemented with supranutritional doses of $\alpha$-T, the density of axo-spinous, asymmetric synapses in hippocampal CA1 stratum radiatum was significantly higher as compared to that found in agematched, control animals $\left(0.70 \pm 0.04 / \mu \mathrm{m}^{2}\right.$ and $0.49 \pm 0.03 / \mu^{2}$ in treated and control rats, respectively; $\mathrm{P}=0.02$; Figure 2 ). Instead, quantitative analyses of basic morphometric features of synapses failed to reveal major between-groups differences: as reported in Table 1, the perimeter of axon terminals, the 
length of postsynaptic specializations and their number per synapse, as well as the linear extension of bouton-spine interfaces were similar in the two groups (Table 1).

Interesting changes were observed in the morphological organization of neuron-glia relationships at hippocampal synapses. As an index reflecting the extension of glial coverage of synapses, we calculated, both for axon terminals and dendritic spines, the ratio between the contour length that was in direct apposition with distal astrocytic processes and the total perimeter (excluding the length of bouton-spine apposition). Between-groups differences were found for axon terminals, with adult offspring from tocopherol-supplemented dams showing significantly higher ratio $(0.25 \pm 0.008$ vs $0.19 \pm 0.013$ in treated and control rats, respectively; $\mathrm{P}=0.02$; Figure $3 \mathrm{~A}$ ), thus indicating a more extended glial coverage of presynaptic boutons at axo-spinous CA1 synapses. In contrast, ratio between glia-surrounded and total perimeter length was unchanged when calculated for postsynaptic spine profiles $(0.15 \pm 0.013$ vs $0.15 \pm 0.011$ in treated and control groups, respectively; $\mathrm{P}=0.98$; Figure $3 \mathrm{~B}$ ).

In addition, possible changes in the proportion of tripartite synapses in CA1 stratum radiatum were assessed by calculating the percentage of synapses contacted by astrocytic endfeet at their bouton-spine junction (thus considering only intrasynaptic glia, directly facing the site of presynaptic release) over the total synapses. This percentage was significantly higher in treated animals respect to controls ( $36 \pm 0.02$ vs $25 \pm 0.02 ; \mathrm{P}=0.03$; Figure $3 \mathrm{C}$ ), thus pointing to a surplus of hippocampal tripartite synapses in adult offspring of $\alpha$-T-supplemented mothers.

\section{Discussion}

As a main outcome of present ultrastructural study, we show that the hippocampus of adult rats born to dams fed with supranutritional doses of $\alpha-T$, as compared to agematched controls, displays: i) higher density of asymmetric (thus excitatory) axo-spinous synapses; ii) larger astrocytic coverage of presynaptic terminals; iii) higher number of axo-spinous synapses contacted by astrocytic endfeet at bouton-spine interface, i.e., tripartite synapses. These anatomical findings extend our previous data obtained in the very same experimental model, showing that maternal dietary loads of $\alpha$-Tocopherol promote permanent deficits in hippocampal synaptic plasticity and spatial learning in adult offspring. ${ }^{8,26}$

In maternally-supplemented rats, modifica-
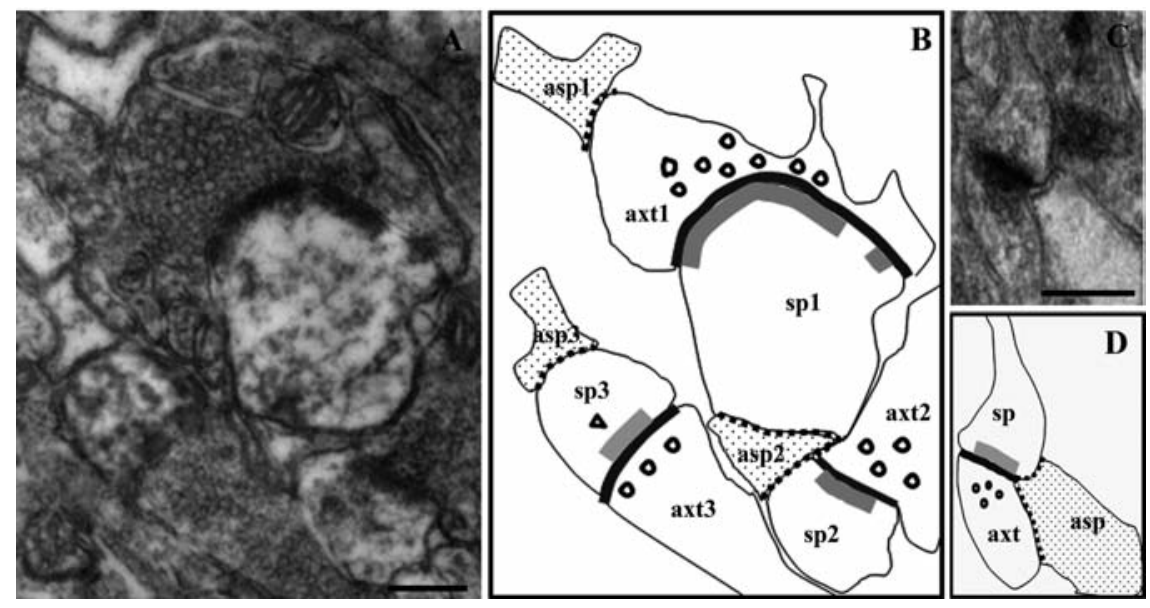

Figure 1. Morphometric analyses of axo-spinous synapses and glia-synapse relationships. Examples of micrographs from CA1 stratum radiatum used for morphometrical analyses, showing axo-spinous synapses surrounded by distal astrocytic processes (A and C). Schematic drawings reproducing the corresponding micrographs are depicted in $B$ and $D$. $A$ and $B$, an axon terminal (axt1) contacted extra-synaptically (i.e. far away from the cleft) by an astrocytic process (asp1), and two dendritic spines (sp1 and sp3) contacted by extra-synaptic glial processes (asp2 and asp3) are shown; asp2, extra-synaptic on sp1, is also in direct apposition with axt $2 / \mathrm{sp} 2$ bouton-spine interface, thus being intra-synaptic on this synapse. In $\mathrm{C}$ and $\mathrm{D}$, a synapse is contacted at its bouton-spine interface by an astrocytic process (intra-synaptic) covering both pre- and post-synaptic profiles. The membrane apposition between neuronal synaptic profiles and glial processes is marked with dotted lines; bouton-spine interfaces (bold black line) and postsynaptic specializations (double bold gray line) are also marked. As an example of morphometric analyses carried out in the study, measures calculated for axt1/sp1 synapse (in B) are as follows: axt1 perimeter, $2396 \mathrm{~nm}$; portion of axt1 profile covered by asp1, $238 \mathrm{~nm}$ (gliacovered/total profile ratio: 0.1 ); $s p 1$ perimeter, $1310 \mathrm{~nm}$; portion of $\mathrm{sp} 1$ profile covered by asp2, $332 \mathrm{~nm}$ (glia-covered/total profile ratio: 0.25 ); length of post-synaptic specializations in sp1, $585 \mathrm{~nm}$ and $67 \mathrm{~nm}$; length of axt1/sp1 bouton-spine apposition, $800 \mathrm{~nm}$. sp, dendritic spine; axt, axon terminal; asp, astrocytic process. Scale bars: A,C) $250 \mathrm{~nm}$.

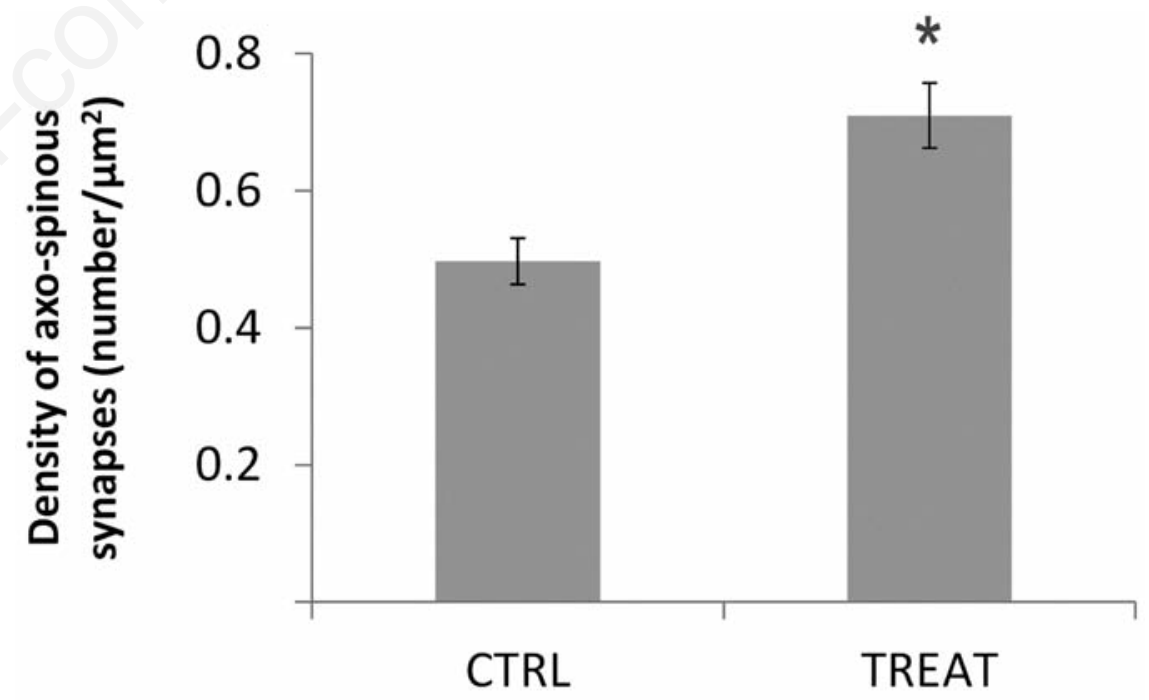

Figure 2. The density of hippocampal synapse is increased in adult offspring from tocopherol-supplemented mothers. Histogram showing the results of quantitative analysis of the density of axo-spinous synapses in CA1 stratum radiatum of adult rats born to mothers fed supranutritional doses of $\alpha$-T (TREAT) and age-matched controls (CTRL). Density is calculated as number of synapses per $\mu^{2}$. Data are presented as mean values \pm SEM. Synapse density is significantly higher in adult rats exposed to $\alpha-T$ during development. Student's t-test; ${ }^{*} \mathbf{P}<0.05$. 
tions of redox state in embryonic and postnatal brain induced by $\alpha$-tocopherol acting as antioxidant might occur, thereby possibly playing a role in promoting the observed ultrastructural, long-lasting effects in adult hippocampus. Endogenous reactive oxygen species (ROS) have been reported to affect proliferation of neural stem/progenitor cells derived from hippocampus of embryonic mice, with antioxidant compounds attenuating the proliferative activity of cultured cells; ${ }^{33}$ moreover, ROS and antioxidant treatment have been shown to influence morphological and physiological differentiation of cortical cultured neurons from embryonic rats ${ }^{34}$ in addition, postnatal treatment (from P2 to P25) with antioxidant compounds, such as NADPH oxidase inhibitors, has been recently shown in rats to decrease the levels of endogenously produced ROS at all developmental ages, in association to macroscopic changes in cerebellar anatomy (i.e., changes in foliation) and alterations in motor behavior at juvenile ages. ${ }^{35} \mathrm{In}$ our previous work, ${ }^{8}$ we failed to detect major effects of maternally-delivered tocopherol on morphological maturation in developing hippocampus. In fact, no gross anatomical alterations in hippocampal structure and layering were observed at any ages; in addition, we found no changes in morphological differentiation of hippocampal pyramidal neurons, whose dendritic extension and complexity at P14 was similar to that measured in untreated, age-matched control rats ${ }^{8}$. Collectively, these findings make unlikely that early developmental changes in redox status could be seen as a major mediating factor in provoking the ultrastructural effects observed in the hippocampus of adult rats maternally-exposed to high doses of $\alpha$-tocopherol.

During brain ontogenesis, overproduction of synapses and their subsequent elimination through activity-dependent processes are critical for correct refinement and normal functioning of neural circuits. ${ }^{36,37}$ Moreover, dynamic processes of assembly and disassembly of synapses persist into adulthood; ${ }^{38,39}$ in rodent hippocampus, including CA1, synapse density has been shown to increase into adult age, ${ }^{40,41}$ nearly doubling between postnatal day 15 and $48,{ }^{42}$ thus pointing to a highly protracted period of synapse formation and refinement. The increase of axo-spinous synapses observed in CA1 stratum radiatum of adult rats maternally exposed to excess of $\alpha$-T may reflect an aberrant gauging of synapse production/elimination balance during hippocampal maturation. We have previously shown that maternal supranutritional intake of $\alpha$-T does not affect developmental expression of important pre- and postsynaptic markers: at P14, in fact, the hippocampal expression level, topographical distribution and cellular localization

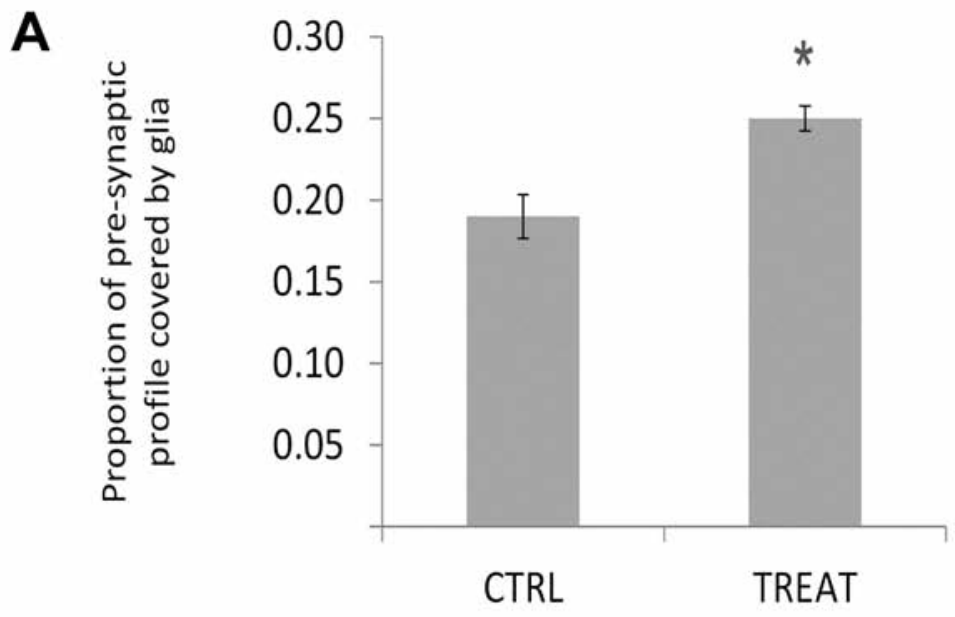

B

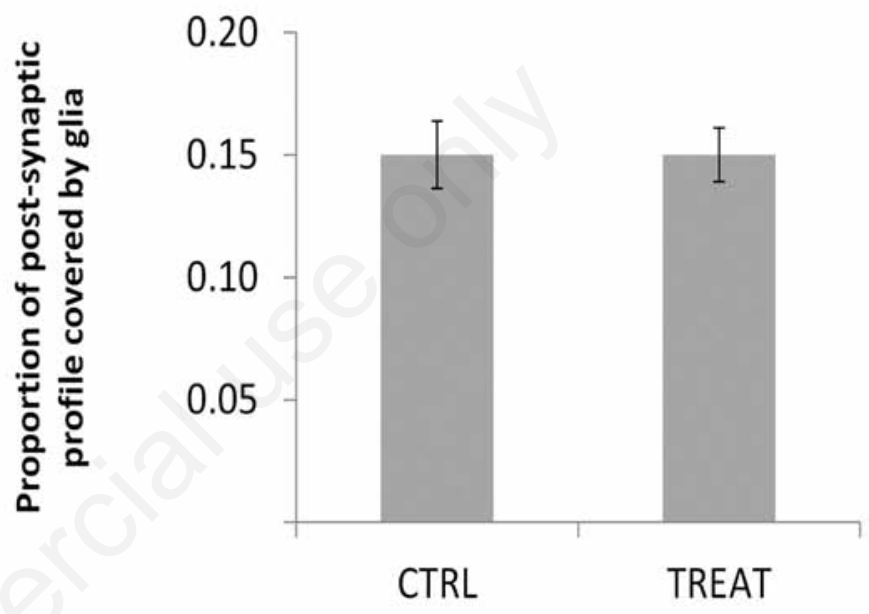

C

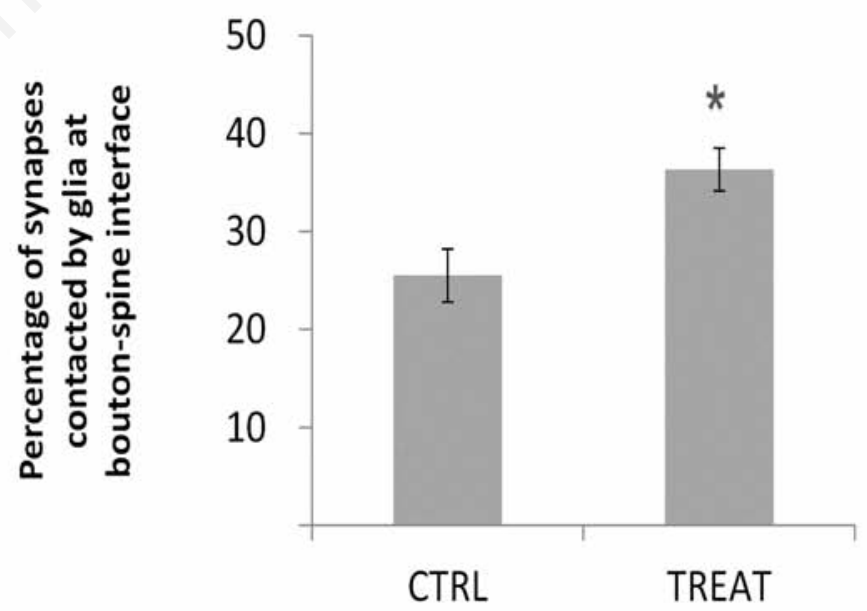

Figure 3. Morphological organization of neuron-glia relationships at hippocampal synapses are affected by maternal loads of $\alpha-T$. Histograms in A and B report the average ratio between the linear portion of axon terminal (A) or dendritic spine (B) profile covered by glia and their total perimeter length (excluding bouton-spine apposition length). For axon terminals (A), this ratio is significantly higher in adult offspring from tocopherol-supplemented dams (TREAT) respect to controls (CTRL), indicating a more extended glial coverage of presynaptic boutons. In contrast, no differences were found for dendritic spines (B). Histogram in $\mathrm{C}$ shows the percentage of axo-spinous synapses contacted by astrocytic endfeet at bouton-spine interface over the total synapses. This percentage is significantly higher in $\alpha$-T-treated animals (TREAT) respect to controls (CTRL), pointing to a relative surplus of tripartite synapses in CA1 of adult offspring of $\alpha$-T-supplemented mothers. Data are presented as mean values \pm SEM. Student's t-test; * $\mathbf{P}<0.05$. 
of synatophysin and spinophilin were virtually normal. ${ }^{8}$ Given that synaptogenesis largely outpaces synapse elimination at early postnatal ages, ${ }^{43}$ a major increment of synapse production rate is unlikely to occur in our model. More plausibly, the increase of synapse density observed in adult offspring from $\alpha$-T-supplemented mothers could be due to reduced synaptic pruning. Maternal loads of tocopherol deeply influence postnatal pattern of hippocampal PKC activity in offspring, depressing PKC phosphorylation and reducing the expression of PKC-dependent, developmental-related proteins. ${ }^{8} \mathrm{~A}$ crucial role for PKC activity in regulating synapse pruning was proposed in studies showing that adult PKC mutant mice display defective elimination of surplus climbing fibers onto cerebellar Purkinje cells, accompanied by motor coordination impairment. ${ }^{19,44}$ Down-regulation of hippocampal PKC phosphorylation levels in tocopherol-treated animals persists into late phases of postnatal maturation, at least up to weaning, ${ }^{8}$ thus possibly affecting synapse elimination processes occurring at juvenile age. Alterations in the establishment, maintenance, or pruning of synapses have been postulated to occur in several neurodevelopmental disorders, including autism, epilepsy, learning disabilities, schizophrenia; ${ }^{45}$ in this context, present findings supporting the view that manipulations of early nutritional environment - as an excessive $\alpha$-T maternal intake and delivery to offspring - can lead to long-lasting changes in synapse density and neural circuits refinement, gain particular relevance.

Maternal dietary loads of $\alpha$-T differentially influence distinct kinds of hippocampusdependent learning in adult offspring, since spatial learning in Morris Water Maze is impaired, whereas contextual fear conditioning is improved. ${ }^{26}$ These two learning types are supported by different extra-hippocampal regions and neural pathways: in particular, contextual fear conditioning requires the integrity of the amygdala, whose substantial projections to various levels of hippocampal formation, including CA1, have been documented in rats. ${ }^{46}$ Although the origin of surplus synapses in CA1 stratum radiatum was not determined, an outgrowth of fibers coming from amygdala could potentiate the amygdaloid input to the hippocampus, thus selectively favoring the improvement of fear conditioning behavior. Such a selective circuit remodeling upon early life nutritional insult may be possibly framed into the general concept of the region-specific morphological and functional adaptative plasticity shown by amygdaloid neurons in response to a variety of environmental and behavioral manipulations. ${ }^{47,48}$

Present results also show that basic mor- phometric features of axo-spinous synapse are not affected by maternal supplementation with supranutritional doses of $\alpha$-T. The perimeter of pre-synaptic axon terminals, the length of postsynaptic specializations and their number per synapse, as well as the extension of bouton-spine contacts were virtually unchanged respect to controls. These findings indicate that hippocampal synapses in adult offspring of tocopherol-supplemented dams, although increased in number, retain normal morphological and geometrical characteristics, thus suggesting that long-term effects on synapse formation/elimination balance are not associated with major alterations of synapse maturational processes. Yet some caution is needed, since structural dimensions and morphometric characteristics of dendritic spines, due to their highly variable shapes, ${ }^{49}$ could not be systematically quantified by single ultrathin sections approach used in our study. Interestingly, major changes were found in glia-synapse anatomical relationships. In adult rats maternally exposed to excessive $\alpha$-T intake, the linear portion of axon terminal profiles that was surrounded by astrocytic processes was more extended as compared to control animals, thus pointing to a heavier glial coverage of presynaptic boutons; in addition, the percentage of synapses that were contacted by astrocytic endfeet at the bouton-spine junction, i.e., the very site of presynaptic neurotransmitter release, was remarkably higher, thus suggesting a surplus of axo-spinous tripartite synapses. ${ }^{50}$ Present anatomical observations indicate that early exposure to supranutritional tocopherol intake can result in permanent alterations of neuron-glia morphological organization at synapses. The concomitant occurrence of synapse outnumber and increased glial ensheathing of synapses is particularly relevant at the light of evidence showing that astrocytes can actively participate in the formation and maintenance of synapses, as well as in controlling synapse turnover required for neural development and plasticity. ${ }^{51-54}$ Adding astroglia into neuronal cultures from rat hippocampus was reported to increase the density of synapses and promote gamma-aminobutyric-acid (GABA) and glutamate receptor clustering, thus facilitating spontaneous network activity; ${ }^{55}$ moreover, studies in organotypic hippocampal cultures have shown that astrocytic contacts play an important role in regulating development of individual synapses, increasing the lifetime of dendritic protrusions and influencing the stability of synaptic connections. ${ }^{56}$

Perisynaptic glia has been recently proposed as integral synaptic element. ${ }^{50,57}$ In fact, perisynaptic astrocytes respond to neurotransmitters released during neuronal activity ${ }^{58,59}$ and release neuroactive substances that can influ- ence neuronal excitability and synaptic transmission; ${ }^{60-62}$ moreover, glia-mediated glutamate uptake via GLAST/GLT-1 transporters regulates glutamate clearance in the cleft and glutamate spillover.63,64 Several mechanisms underlying astrocytic effects on synaptic plasticity have been described in diverse brain regions including hippocampus, having either facilitating or inhibiting, context-dependent roles: glia-derived transmitters, such as ATP/adenosine or D-serine, can modulate glutamate receptor activity and trafficking, ${ }^{65-68}$ while glial transport influences temporal and spatial patterns of NMDA receptor activation by controlling glutamate concentration at synapse. ${ }^{69,70}$ At the light of this evidence, alterations in perisynaptic glial coverage and tripartite synapse function are likely to influence synaptic plasticity and behavioral learning performance. It is thus tempting to speculate that the increased astrocytic coverage of CA1 stratum radiatum synapses could contribute to the impairment of LTP induction and alterations in hippocampus-dependent learning abilities previously reported in the same model of adult rats born to $\alpha$-T-supplemented mothers. ${ }^{8,26}$ Recent findings seem in contrast with this view: in transgenic mice expressing an inositol-triphosphate absorbent that attenuates astrocytic calcium signaling, behavioral deficits in hippocampus-dependent spatial and contextual fear memory were associated with a reduction, instead of an increase, of glial coverage at CA1 asymmetric synapses. ${ }^{71}$ However, given the fundamental role played by intracellular calcium transients in glial physiology and excitability, ${ }^{72,73}$ blunting calcium signaling is supposed to affect most, if not all, aspects of activity-related astrocytic functions, thus deeply and globally disrupting neuron-glia communication processes. In our model, the increased glial ensheathing of synapses might potentially affect LTP induction mainly by enhancing glutamate uptake capacity. Recent evidence gives support to this view. Ceftriaxone-mediated enhancement of GLT-1 gene transcription changes GLT-1 expression and distribution, and impairs activity-dependent synaptic plasticity at mossy fibre-CA3 hippocampal synapses.$^{74}$ Moreover, knockout mice lacking Ephrin-A3, a molecule involved in neuron-glia communication processes regulating synapse morphology and function in the hippocampus, ${ }^{75}$ display impaired LTP induction and deficits in hippocampus-dependent tasks, mainly ascribed to post-translational up-regulation of glutamate transporters in astrocytes and more efficient glutamate removal from the cleft. $^{70,76}$

In conclusion, adult offspring born to dams that were fed with supranutritional doses of $\alpha$ $\mathrm{T}$ over pregnancy and lactation display permanent hippocampal changes in axo-spinous 
synapse density and in neuron-glia morphological relationships at synapses, together with deficits in LTP induction and spatial memory. These findings confirm and extend previous evidence supporting the general concept that early life environmental stress, including those deriving from nutritional insults, can lead to long-lasting effects in adult brain. It is worth noting that the increase in vitamin $\mathrm{E}$ doses used for pregnant women in most supplementation trials, ranges $40-100$ times the standard recommendation, ${ }^{15,18,77}$ which is comparable to the increase applied to the nutritional intervention of this study. Therefore, present results emphasize the need to carefully evaluate the safety of developmental exposure to high doses of $\alpha$-T in animal species and particularly in humans.

\section{References}

1. Azzi A. Molecular mechanisms of $\alpha$-tocopherol action. Free Radic Biol Med 2007;43:16-21.

2. Azzi A, Gysin R, Kempná P, Munteanu A, Negis Y, Villacorta L, et al. Vitamin E mediates cell signaling and regulation of gene expression. Ann NY Acad Sci 2004;1031: 86-95.

3. Rimbach G, Fisher A, Stoecklin E, Barella L. Modulation of hepatic gene expression by alpha tocopherol in cultured cells and in vivo. Ann NY Acad Sci 2004;1031:102-108.

4. Galli F, Azzi, A. Present trends in vitamin E research. Biofactors 2010;36:33-42.

5. Brigelius-Flohe R, Kelly FJ, Salonen JT, Neuzil J, Zingg JM, Azzi A. The European perspective on Vitamin E: current knowledge and future research. Am J Clin Nutr 2002;76:703-716.

6. Betti M, Minelli A, Canonico B, Castaldo P, Magi S, Aisa MC, et al. Antiproliferative effects of tocopherols (vitamin E) on murine glioma C6 cells: homologue-specific control of PKC/ERK and cyclin signaling. Free Radic Biol Med 2006;41:464-72.

7. Varga Z, Kosaras E, Komodi E, Katko M, Karpati I, Balla J, et al. Effects of tocopherols and 2,2'-carboxyethyl hydroxychromans on phorbol-ester-stimulated neutrophils. J Nutr Biochem 2008;19:320-7.

8. Betti M, Ambrogini P, Minelli A, Floridi A, Lattanzi D, Ciuffoli S, et al. Maternal dietary loads of $\alpha$-tocopherol depress protein kinase $\mathrm{C}$ signaling and synaptic plasticity in rat postnatal developing hippocampus and promote permanent deficits in adult offspring. J Nutr Biochem 2011;22: 60-70.

9. Ferri P, Cecchini T, Ambrogini P, Betti M, Cuppini R, Del Grande P, et al. $\alpha$-toco- pherol affects neuronal plasticity in adult rat dentate gyrus: the possible role of PKC $\alpha$. J Neurobiol 2006;66:793-810.

10. Eriksson UJ, Borg LA. Diabetes and embryonic malformations: role of substrateinduced free-oxygen radical production for dysmorphogenesis in cultured rat embryos. Diabetes 1993;42:411-9.

11. Mikhail MS, Anyaegbunam A, Garfinkel D, Palan PR, Basu J, Romney SL. Preeclampsia and antioxidant nutrients: decreased plasma levels of reduced ascorbic acid, $\alpha$-tocopherol and beta-carotene in women with preeclampsia. Am J Obst Gynecol 1994;171:150-7.

12. Von Mandach U, Huch R, Huch A. Maternal and cord serum vitamin $\mathrm{E}$ levels in normal and abnormal pregnancy. Int J Vitam Nutr 1993;63:26-32.

13. Gupta S, Aziz N, Sekhon L, Agarwal R, Mansour G, Li J. Lipid peroxidation and antioxidant status in preeclampsia: a systematic review. Obst Gynecol Surv 2009;64:750-9.

14. Gallo C, Renzi P, Loizzo A, Piacente S, Festa M, Caputo M, et al. Potential therapeutic effects of vitamin $\mathrm{E}$ and $\mathrm{C}$ on placental oxidative stress induced by nicotine: an in vitro evidence. Open Biochem 2010;4:77-82

15. Rumbold AR, Crowther CA. Vitamin E supplementation in pregnancy. Cochrane database Syst Rev 2005;18:CD004069.

16. Poston L, Briley AL, Seed PT, Kelly FJ, Shennan AH. Vitamins in Pre-eclampsia (VIP) Trial Consortium Vitamin C and Vitamin E in pregnant women at risk for pre-eclampsia (VIP trial): randomized placebo-controlled trial. Lancet 2006;367: 1145-54.

17. Polyzos NP, Mauri D, Tsappi M, Tzioras S, Kamposioras K, Cortinovis I. Combined Vitamin $\mathrm{C}$ and $\mathrm{E}$ supplementation during pregnancy for pre-eclampsia prevention: a systematic review. Obst Gynecol Surv 2007;62:202-6.

18. Rumbold AR, Duley L, Crowther CA, Hasiam RR. Antioxidants for preventing pre-eclampsia. Cochrane Database Syst Rev 2008;23:CD004227.

19. Kano M, Hashimoto K, Chen C, Abeliovich A, Alba A, Kurihara $\mathrm{H}$, et al. Impaired synapse elimination during cerebellar development in PKC $\alpha$ mutant mice. Cell 1995;83:1223-31.

20. Hama H, Hara H, Yamaguchi K, Miyawaki A. PKC signaling mediates global enhancement of excitatory synaptogenesis in neurons triggered by local contact with astrocytes. Neuron 2004;41:405-15.

21. Kapfhammer JP. Cellular and molecular control of dendritic growth and development of cerebellar Purkinje cells. Prog
Histochem Cytochem 2004;39:131-82.

22. Shahak H, Slotkin TA, Yanai J. Alterations in PKCgamma in the mouse hippocampus after prenatal exposure to heroin: a link from cell signaling to behavioral outcome. Brain Res Dev Brain Res 2003;140:117-25.

23. Xu SZ, Bullock L, Shan CJ, Cornelius K, Rajanna B. PKC isoforms were reduced by lead in the developing rat brain. Int J Dev Neurosci 2005;23:53-64.

24. Paulraj R, Behari J. Protein kinase C activity in the developing rat brain cells exposed to $2.45 \mathrm{GHz}$ radiation. Electromagn Biol Med 2006;25:61-70.

25. Yanai J, Brick-Turin Y, Dotan S, Langford R, Pinkas A, Slotkin TA. A mechanism-based complementary screening approach for the amelioration and reversal of neurobehavioral teratogenicity. Neurotoxicol Teratol 2010;32:109-13.

26. Ambrogini P, Ciuffoli S, Lattanzi D, Minelli A, Bucherelli C, Baldi E, et al. Maternal dietary loads of $\alpha$-tocopherol differentially influence fear conditioning and spatial learning in adult offspring. Physiol Beha 2011;104:809-15.

27. DeFelipe J, Conti F, Van Eyck SL, Manzoni T. Demonstration of glutamate-positive axon terminals forming asymmetric synapses in cat neocortex. Brain Res 1988;455:162-5.

28. Jarrard LE. On the role of the hippocampus in learning and memory in the rat. Behav Neural Biol 1993;60:9-26.

29. Mizuno K, Giese KP. Hippocampusdependent memory formation: do memory type-specific mechanisms exist? J Pharmacol Sci 2005;98:191-7.

30. Minelli A, Castaldo P, Gobbi P, Salucci S, Magi S, Amoroso S. Cellular and subcellular localization of $\mathrm{Na}+-\mathrm{Ca} 2+$ exchanger protein isoforms, NCX1, NCX2, and NCX3 in cerebral cortex and hippocampus of adult rat. Cell Calcium 2007;41:221-34.

31. Gobbi P, Castaldo P, Minelli A, Salucci S, Magi S, Corcione E, et al. Mitochondrial localization of $\mathrm{Na}+/ \mathrm{Ca} 2+$ exchangers NCX1-3 in neurons and astrocytes of adult rat brain in situ. Pharmacol Res 2007;56: 556-65.

32. Peters A, Palay SL, Webster HdeF. The fine structure of the nervous system. Neurons and their supporting cells, 3th ed. New York: Oxford University Press, 1991.

33. Yoneyama M, Kawada K, Gotoh Y, Shiba T, Ogita K. Endogenous reactive oxygen species are essential for proliferation of neural stem/progenitor cells. Neurochem Int 2010;56:740-6.

34. Tsatmali M, Walcott EC, Makarenkova H, Crossin KL. Reactive oxygen species modulate the differentiation of neurons in clonal cortical cultures. Mol Cell Neurosci 
2006;33:345-57.

35. Coyoy A, Olguin-Albuerne M, MartinezBriseno P, Moran J. Role of reactive oxygen species and NADPH-oxidase in the development of rat cerebellum. Neurochem Int 2013;62:998-1011.

36. Munno DW, Syed NI. Synaptogenesis in the CNS: an odyssey from wiring together to firing together. J Physiol 2003;552:1-11.

37. Hua JY, Smith SJ. Neural activity and the dynamics of central nervous system development. Nat Neurosci 2004;7:327-32.

38. Chklovskii DB, Mel BW, Svoboda K. Cortical rewiring and information storage. Nature 2004;431:782-8.

39. De Roo M, Klauser P, Garcia PM, Poglia L, Muller D. Spine dynamics and synapse remodeling during LTP and memory processes. Prog Brain Res 2008;169:199207.

40. Harris KM, Jensen FE, Tsao B. Threedimensional structure of dendritic spines and synapses in rat hippocampus (CA1) at postnatal day 15 and adult ages: implications for the maturation of synaptic physiology and long-term potentiation. J Neurosci 1992;12:2685-705.

41. Eastwood SL, Weickert CS, Webster MJ, Herman MM, Kleinman JE, Harrison PJ. Synaptophysin protein and mRNA expression in the human hippocampal formation from birth to old age. Hippocampus 2006;16:645-54.

42. Pokorny J, Yamamoto T. Postnatal ontogenesis of hippocampal CAl area in rats. II. Development of ultrastructure in stratum lacunosum and moleculare. Brain Res Bull 1981;7:121-30.

43. Garner CC, Waites CL, Ziv NE. Synapse development: still looking for the forest, still lost in the trees. Cell Tissue Res 2006;326:249-62.

44. Chen C, Kano M, Abeliovich A, Chen L, Bao S, Kim JJ, et al. Impaired motor coordination correlates with persistent multiple climbing fiber innervation in PKC $\alpha$ mutant mice. Cell 1995;83:1233-42.

45. Paus T, Keshavan M, Giedd JN. Why do many psychiatric disorders emerge during adolescence? Nat Rev Neurosci 2008;9: 947-57.

46. Pikkarainen M, Rönkkö S, Savander V, Insausti R, Pitkänen A. Projections from the lateral, basal, and accessory basal nuclei of the amygdala to the hippocampal formation in rat. J Comp Neurol 1999;403: 229-60.

47. McEwen BS. The ever-changing brain: cellular and molecular mechanisms for the effects of stressful experiences. Dev
Neurobiol 2012;72:878-90.

48. Davidson RJ, McEwen BS. Social influences on neuroplasticity: stress and interventions to promote well-being. Nat Neurosci 2012;15:689-95.

49. Harris KM, Stevens JK. Dendritic spines of CAl pyramidal cells in the rat hippocampus: serial electron microscopy with reference to their biophysical characteristics. J Neurosci 1989;9:2982-97.

50. Perea G, Navarrete M, Araque A. Tripartite synapses: astrocytes process and control synaptic information. Trends Neurosci 2009;32:421-31.

51. Mauch DH, Nägler $K$, Schumacher $S$, Göritz C, Müller EC, Otto A, et al. CNS synaptogenesis promoted by glia-derived cholesterol. Science 2001;294:1354-7.

52. Allen NJ, Barres B. Signaling between glia and neurons: focus on synaptic plasticity. Curr Opin Neurobiol 2005;15:542-8.

53. Pfrieger FW. Role of glial cells in the formation and maintenance of synapses. Brain Res Rev 2010;63:39-46.

54. Eroglu C, Barres B. Regulation of synaptic connectivity by glia. Nature 2010;468:22354.

55. Boehler MD, Wheeler BC, Brewer GJ. Added astroglia promote greater synapse density and higher activity in neuronal networks. Neuron Glia Biol 2007;3:127-40.

56. Nishida H, Okabe S. Direct astrocytic contacts regulate local maturation of dendritic spines. J Neurosci 2007;27:331-40.

57. Perea G, Araque A. Glia modulates synaptic transmission. Brain Res Rev 2010;63:93102.

58. Schipke CG, Kettenmann H. Astrocyte responses to neuronal activity. Glia 2004; 47:226-32.

59. Perea G, Araque A. Properties of synaptically-evoked astrocyte calcium signal reveal synaptic information processing by astrocytes. J Neurosci 2005;25:2192-203.

60. Volterra A, Steinhauser C. Glial modulation of synaptic transmission in the hippocampus. Glia 2004;47:249-57.

61. Kozlov AS, Angulo MC, Audinat E, Charpak $\mathrm{S}$. Target cell-specific modulation of neuronal activity by astrocytes. Proc Natl Acad Sci USA 2006;103:10058-63.

62. Parpura V, Zorec R. Gliotransmission: exocytotic release from astrocytes. Brain Res Rev 2010;63:83-92.

63. Diamond JS. Deriving the glutamate clearance time course from transporter currents in CA1 hippocampal astrocytes: transmitter uptake gets faster during development. J Neurosci 2005;25:2906-16.

64. Tsingounis AV, Wadiche JI. Glutamate transporters: confining runaway excitation by shaping synaptic transmission. Nat Rev Neurosci 2007;8:935-47.

65. Gordon GR, Baimoukhametova DV, Hewitt SA, Rajapaksha WR, Fisher TE, Bains JS. Norepinephrine triggers release of glial ATP to increase postsynaptic efficacy. Nat Neurosci 2005;8:1078-86.

66. Pascual 0, Casper KB, Kubera C, Zhang J, Revilla-Sanchez R, Sul JY, et al. Astrocytic purinergic signaling coordinated synaptic networks. Science 2005;310:113-6.

67. Panatier A, Theodosis DT, Mothet JP, Touquet B, Pollegioni L, Poulain DA, et al. Glia-derived D-serine controls NMDA receptor activity and synaptic memory. Cell 2006;125:775-84.

68. Henneberger C, Papouin T, Oliet SH, Rusakov DA. Long-term potentiation depends on release of D-serine from astrocytes. Nature 2010;463:232-6.

69. Katagiri H, Tanaka K, Manabe T. Requirement of appropriate glutamate concentrations in the synaptic cleft for hippocampal LTP induction. Eur J Neurosci 2001;14:547-53.

70. Filosa A, Paixao S, Honsek SD, Carmona MA, Becker L, Feddersen B, et al. Neuronglia communication via EphA4/ephrin-A3 modulates LTP through glial glutamate transport. Nat Neurosci 2009;12:1285-92.

71. Tanaka M, Shih PY, Gomi H, Yoshida T, Nakai J, Ando R, et al. Astrocytic Ca2+ signals are required for the functional integrity of tripartite synapses. Molecular Brain 2013;6:6-18.

72. Verkhratsky A, Orkland RK, Kettenmann H. Glial calcium: homeostasis and signaling function. Physiol Rev 1998;78:99-141.

73. Agulhon C, Petravicz J, McMullen AB, Sweger EJ, Minton SK, Taves SR, et al. What is the role of astrocyte calcium in neurophysiology? Neuron 2008;59:932-46.

74. Omrani A, Melone M, Bellesi M, Safiulina V, Aida T, Tanaka K, et al. Up-regulation of GLT-1 severely impairs LTD at mossy fibreCA3 synapses. J Physiol 2009;587:4575-88.

75. Hruska M, Dalva MB. Ephrin regulation of synapse formation, function and plasticity. Mol Cell Neurosci 2012;50:35-44.

76. Carmona MA, Murai KK, Wang L, Roberts AJ, Pasquale EB. Glial ephrin-A3 regulates hippocampal dendritic spine morphology and glutamate transport. Proc Natl Acad Sci USA 2009;106:12524-9.

77. Boskovic R, Gargaun L, Oren D, Djulus J, Koen G. Pregnancy outcome following high doses of vitamin E supplementation. Reprod Toxicol 2005;20:85-8. 